\title{
Intelligent Home Automation and Environment Creation Using Arm9
}

\author{
Chetana Naresh Nemade ${ }^{1}$, M. D. Bagde ${ }^{2}$ \\ ${ }^{1}$ G. H. Raisoni college of Engineering, Jalgaon \\ ${ }^{2}$ G.H. Raisoni College of Engineering, Department of Electronics, Jalgaon
}

\begin{abstract}
This paper represents the design and implementation of smart surveillance monitoring system and mood detection using ARM9 and MATLAB for industrial applications. Usually this project is implemented using ARM9.Here, system design to provide essential security and energy conservation to our industrial and for other control applications. ARM9 affordable for large scale industries but we are providing solution to if for small scale industries using microcontroller.
\end{abstract}

Keyword: Facial expression, PIR sensor, Webcam, Light sensor

\section{Introduction}

In this paper, microcontroller senses input through all the sensors like temperature, light sensor etc. and accordingly controls the parameters like heater and fan.Then set/control the current and saved values are displayed using $16 * 2 \mathrm{LCD}$ display. The sensor gathers the values and sends the information to the microcontroller continuously. The microcontrollers receive and send the corresponding values to the LCD to display. This is a cyclic process which performs till our requirement is done.ARM9 operates and controls the motion detectors and also use image processing (MATLAB) video cameras for remote sensing, surveillance, streams live video and records it for future playback. This project is hardware as well as software based.

Home automation technology is emerged with the aim to facilitate activities in the household or home, and provide a more comfortable life for residence. Example tasks include programming the TV set, opening/closing window blinds, or controlling a home environment system. However, there are no user interfaces standards for these devices, frequently result in greater complexity of use. The same technology that simplifies life by enabling a greater number of features in single devices can also complicate daily lives, it create more difficult to learn and use this technology. In this paper the daily energy consumption of a single home that acts as the consumer and producer of electrical energy Power consumption patterns of house appliances vary depending on the hour of the day.

Emotions are associated with human feelings and behavior, which in turn are related to daily activities. Facial expressions are synchronizing the dialogue means brow rising and nodding. Interpret the content and intent by means of lip reading, blinking, signal comprehension, disagreement, and convey message about cognitive, psychological, and affective states.

\section{Methodology}

Here, detect the Mood of user as well as human enters the room. Facial expression is recognized as $\mathrm{SAD} / \mathrm{HAPPY}$ /ANGRY. Facial feature point tracking which is step in emotion detection. One of the most common methods-Detects the faces using a Viola-Jones face detector.

Method of all detections shown in figure1

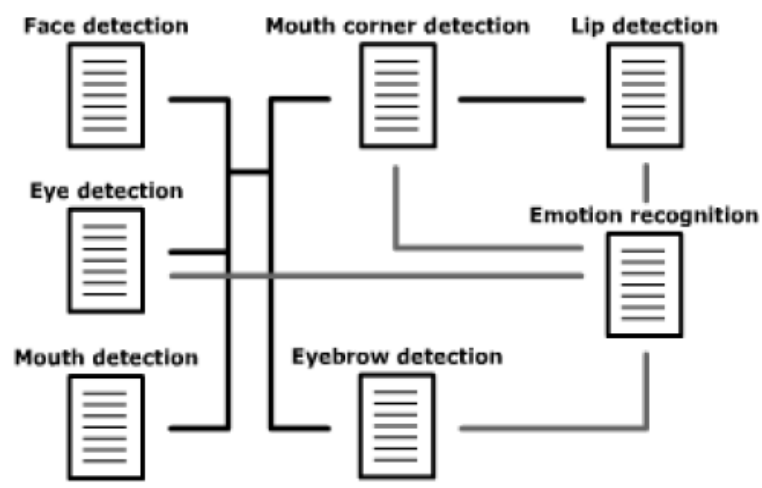

Figure 1: Structure of all detected facial features

\subsection{Face Detection}

In this method, before detects the specified feature points, regions of interest have to be set to ensure a smaller overhead and rule out most false positives. To decide the position of the eyes use the same algorithm with a different classifier in the correct the regions of interest which are the upper left and upper right part of the known face region.

\subsection{Eyebrow Detection}

Eyebrow tracker is based on edges using canny edge detection. A mask representing the average shape of an eyebrow is used to find the best candidate.After run canny edge detection on the region of interest above the eye, the mask slides top to bottom to find the most edge withinits shape. The mask is drawn with an angle which is calculated based on the position of the eye. Average is also added to reduce false positives. This method is less robust when used in worst conditions, but performs well in a constrained environment. 


\subsection{Mouth Detection}

Similar to the face detection, locate the mouth based on haarlike features. To detect the corners of the mouth however, use the technique based on edges using canny edge detection and masks. Here, use two different masks representing the corners of the mouth, and the move them top to bottom while calculating how many positive bits are found within the specific region. The same method is again used to find the upper and lower lip. Use the corners of the mouth, which are known, as starting and ending point of our lip masks.

\subsection{Emotion Detection}

Although most existing methods covers learning stages, we implemented an easy yet pretty effective method based on proportions. The distances below are scaled to the current face scale, which define using the distance between both eye;

1) Distance between eyes and eyebrows

2) Distance between eyes and mouth corners

3) Distance between upper and lower lip

\section{Conclusion}

Paper deals with the home automation and detection of mood using different sensors like temperature and light sensors. For example temperature sensor detects the increase in temperature above the set value. Temperature sensor maintains a specific temperature. When the light intensity is lower than the set point then only the lights are turned ON. This way we are reducing the energy consumption and wastage of energy.

\section{References}

[1] J.Cohnand P. Ekman, "Measuring facial action by manual coding, facial EMG, and automatic facial image analysis," 2005.

[2] M. P. D. Vukadinovic, "Fully automatic facial feature point detection using Gabor feature based boosted classifiers," IEEE International Conference on Systems, 2005.

[3] M. Pantic and M. Bartlett, "Machine analysis of facial expressions," inFace Recognition, K. Delac and M. Grgic, Eds. Vienna, Austria: I-Tech Educ. Publishing, 2007

[4] D.M.Han and J.H. Lim," Smart home energy management system using IEEE802.15.4 and ZigBee,"Aug.2010.

[5] Gaglio, S.,LoRe.G, Morana, M and Ortolani, M ()2013, "Gesture Recognition for Improved User Experience in a Smart Environment". 